\title{
EPIG-Seq: extracting patterns and identifying co-expressed genes from RNA-Seq data
}

\author{
Jianying $\mathrm{Li}^{1,2,4}$ and Pierre R. Bushel $\mathrm{l}^{2,3^{*}}$
}

\begin{abstract}
Background: RNA sequencing (RNA-Seq) measures genome-wide gene expression. RNA-Seq data is count-based rendering normal distribution models for analysis inappropriate. Normalization of RNA-Seq data to transform the data has limitations which can adversely impact the analysis. Furthermore, there are a few count-based methods for analysis of RNA-Seq data but they are essentially for pairwise analysis of treatment groups or multiclasses but not pattern-based to identify co-expressed genes.

Results: We adapted our extracting patterns and identifying genes methodology for RNA-Seq (EPIG-Seq) count data. The software uses count-based correlation to measure similarity between genes, quasi-Poisson modelling to estimate dispersion in the data and a location parameter to indicate magnitude of differential expression. EPIG-Seq is different than any other software currently available for pattern analysis of RNA-Seq data in that EPIG-Seq 1) uses count level data and supports cases of inflated zeros, 2) identifies statistically significant clusters of genes that are co-expressed across experimental conditions, 3) takes into account dispersion in the replicate data and 4) provides reliable results even with small sample sizes. EPIG-Seq operates in two steps: 1) extract the pattern profiles from data as seeds for clustering co-expressed genes and 2) cluster the genes to the pattern seeds and compute statistical significance of the pattern of co-expressed genes. EPIG-Seq provides a table of the genes with bootstrapped p-values and profile plots of the patterns of co-expressed genes. In addition, EPIG-Seq provides a heat map and principal component dimension reduction plot of the clustered genes as visual aids. We demonstrate the utility of EPIG-Seq through the analysis of toxicogenomics and cancer data sets to identify biologically relevant co-expressed genes. EPIG-Seq is available at: sourceforge.net/projects/epig-seq.

Conclusions: EPIG-Seq is unlike any other software currently available for pattern analysis of RNA-Seq count level data across experimental groups. Using the EPIG-Seq software to analyze RNA-Seq count data across biological conditions permits the ability to extract biologically meaningful co-expressed genes associated with coordinated regulation.
\end{abstract}

Keywords: EPIG-Seq, Gene expression, RNA-Seq, Clustering, Pattern analysis, Toxicogenomics, Cancer

\section{Background}

The advantages of RNA-sequencing (RNA-Seq) over microarray technology to measure gene expression have been reported recently [1-3]. Methods have been developed to analyze RNA-Seq data based on normalization

\footnotetext{
* Correspondence: bushel@niehs.nih.gov

${ }^{2}$ Microarray and Genome Informatics Group, National Institute of Environmental Health Sciences, Research Triangle Park, NC 27709, USA

${ }^{3}$ Biostatistics and Computational Biology Branch, National Institute of Environmental Health Sciences, 111 T.W. Alexander Drive, P.O. Box 12233, Research Triangle Park, NC 27709, USA

Full list of author information is available at the end of the article
}

of read counts or using raw count data [4-6]. Advantages of normalization are that it adjusts the data according to sequencing library size, accounts for the length of transcripts and allows for the use of analysis tools originally designed for microarray data. However, normalized RNA-Seq data or transformation of count data has limitations [7-10] which can adversely impact the analysis. Alternatively, using raw read counts circumvents the shortcomings of normalization but requires modelling of the data to estimate dispersion, accounting for library size and filtering to avoid cases of inflated zeros. In particular, statistical models of count 
data based on Poisson, beta- or negative-binomial distributions can be severely impacted by outliers in the data [11-13]. Unfortunately, there is a paucity of methodologies that can identify correlated gene expression patterns from RNA-Seq count data across biological conditions (i.e., time course, dose response, factorial study designs) [14]. Such paucity also limits the ability to crossexamine RNA-Seq and microarray analysis through comparable statistical measures, which can lead to discrepancies in data interpretation between these techniques.

We adapted the extracting patterns and identifying coexpressed genes (EPIG) methodology [15] for the identification of co-expressed genes from RNA-Seq data (EPIG-Seq). In the EPIG-Seq software patterns of gene expression across experimental groups are determined using a similarity measure for count data [16] to ascertain correlation between expression profiles, a quasiPoisson model [13] to estimate dispersion in the data and a location parameter as a measure of the magnitude of difference between experimental conditions and control/baseline. EPIG-Seq then clusters each gene expression profile to the pattern for which it has the highest correlation. EPIG-Seq is different than any other software currently available for pattern analysis of RNA-Seq data in that EPIG-Seq identifies statistically significant clusters of co-expressed genes using count level data with or without inflated zeros. Furthermore, EPIG-Seq provides reliable results by taking into account dispersion in the data and defaulting to a robust/non-parametric magnitude of fold change estimator when sample sizes are small. We demonstrate the utility of EPIG-Seq by analyzing publicly available RNA-Seq data sets from the SEquence Quality Control (SEQC) toxicogenomics [1] arm of the MicroArray Quality Control (MAQC) consortium and from The Cancer Genome Atlas (TCGA) [17] breast cancer data portal. Using EPIG-Seq we identify several coexpressed genes related to modes of action (MOAs) of the chemical agents in the toxicogenomics data set and we also extract co-expressed genes that are being explored as molecular targets for breast cancer. Finally, EPIG-Seq has a user-friendly interface, it is also platform independent and provides a heat map, pattern profile plots and a principal component analysis dimension reduction plot of the clustered co-expressed genes as visual aids.

\section{Implementation}

\section{Correlation}

A compiled RNA-Seq gene expression data set consists of a 2-dimensional matrix where each row represents a gene expression profile and each column represents a sample. We denote $x_{i j}$ as the count of reads from sample $j$ mapped to gene $i$ and $x_{k j}$ as the count of reads from sample $j$ mapped to gene $k$. To measure the count level correlation between two gene profiles, EPIG-Seq uses the $C Y_{s}$ similarity measure for count data previously defined as:

$$
C Y_{s_{i k}}=1-\frac{\text { observed } C Y_{d_{i k}}}{\text { maximum } C Y_{d_{i k}}}
$$

where

observed $C Y_{d_{i k}}=\sum_{j=1}^{a}\left(\frac{\left(x_{i j}+x_{k j}\right) \log \left(\frac{x_{i j}+x_{k j}}{2}\right)-x_{i j} \log x_{k j}-x_{k j} \log x_{i j}}{x_{i j}+x_{k j}}\right)$,

$a$ is the total number of samples with read counts mapped to either profile and log is the natural logarithm. As such, $C Y_{s_{i k}}=0$ when two profiles are totally different and $C Y_{s_{i k}}=1$ when the two are identical. The $C Y_{s}$ similarity measure is similar to other distance or similarity metric (i.e., Horn's index [18]) and was originally used for assessing variation in species abundance in ecological and environmental monitoring. Its nomenclature originates from initials of the lead author introducing its use and is shown to outperform other similarity measures on species abundance count data $[16,19]$. CYs works better than the Spearman rank correlation coefficient when the expression of the genes within all the groups is relatively the same except in the control/baseline/reference. The Spearman rank correlation coefficient treats these as ties and hence does not allow responsive but invariant patterns across treatment groups to be extracted. Further details of the computation of the $C Y_{s}$ correlation including the maximization of $C Y_{d}$ (the dissimilarity measure) are available in the Additional file 1.

\section{Magnitude of change}

In EPIG-Seq, the strength of a gene expression profile's signal is defined according to the value of the teststatistic location parameter obtained from a Wilcoxon rank sum non-parametric test [20] measuring the difference between the ranks of the expression of the genes in sample $X$ vs those in sample $Y$. Here, sample $X$ is the biological replicates from the treated, perturbed or diseased group and sample $Y$ is the biological replicates from the controls. When the sample size for each group is small, the approximated Z-statistic from the Wilcoxon rank sum test can be spurious. In such a case, EPIG-Seq defaults to measure the strength of the gth gene's differential expression according to the value of the HodgesLehmann location parameter estimator $\hat{\Delta}_{g}$ for the difference between two groups of independent samples [20]. See the Additional file 1 for further details of the magnitude of change. 


\section{Dispersion}

For each gth gene expression profile, EPIG-Seq estimates the dispersion parameter $\theta$ using a quasi-Poisson regression to model the data. The quasi-Poisson likelihood model is commonly used for overdispersed count data as it incorporates $\theta$ into the Poisson model such that that $\mathrm{V}\left(Y_{g}\right)=\mu_{g} \theta_{g}$ [21]. Further details of the dispersion and count data modelling are available in the Additional file 1.

\section{Clustering of gene expression profiles to patterns}

EPIG-Seq runs in two steps. The first step (pattern profile determination) involves pairwise correlations of all the genes and tallying those which have a $\mathrm{CY}_{\mathrm{s}}$ correlation $>=$ Rt1 and at least Mt-1 highly correlated genes. The genes that meet these criteria are further filtered according to those with a magnitude of change $\leq$ St1 and dispersion $<$ or $>5 \%$ in each tail of the distribution. The remaining genes are defined as the pattern profiles for co-expression clustering. In step two (clustering of the genes to the pattern profiles), genes are clustered to the patterns using initialization and recursion strategies that are typical in standard clustering methodologies [22, 23]. The $C Y_{s_{i k}}$ measure is used to correlate the $i$ th gene to the $k$ th pattern profile. The gene is assigned to the pattern to which it has the highest similarity to (i.e. a $\mathrm{CY}_{\mathrm{s}}$ correlation $>=\mathrm{Rt} 2$ ). Once all the genes are assigned, a representative gene for each pattern is determined by a pattern correlation score (PCS) and the clustering continues recursively until there are no more movement of genes or the \# of moves $=100$. See the Additional file 1 for further details of the PCS, the clustering of gene expression profiles to patterns and the EPIG-Seq algorithm pseudo code.

\section{Assessing the significance of the clustering}

To assess the significance of the clustering of the coexpressed genes to the extracted patterns, we performed $B$ number of bootstrapped assignments of $P$ random gene profiles to a pattern and compute the PCS each time to compare to the observed PCS for that pattern. Briefly, for $B$ times and for a given pattern containing $P$ gene profiles, we randomly select $P$ number of gene profiles from the data set. Then, for the selected $P$ random profiles, we compute the bootstrapped PCS. The $p$-value for a pattern is computed as the number $(n)$ of times one of these bootstrapped scores is greater than the observed score. Thus, $p$-value $=n / B$. Statistical significance of each co-expressed gene is determined by the $p$-value from the generalized linear model of the count data. The resulting patterns represent statistically significant clusters of genes that are biologically meaningful due to their shared co-expression across the treatment groups/ biological conditions. In other words, the genes respond similarly. See the Additional file 1 for further details of the count data modelling of RNA-Seq data.

\section{Publicly available data TCGA breast cancer RNA-Seq data}

The Cancer Genome Atlas (TCGA) provides open access to genomic data acquired from various forms of cancer. Institutional review boards at each tissue source site reviewed protocols and consent documentation and approved submission of cases to TCGA. Cases were staged according to the American Joint Committee on Cancer (AJCC) staging system. To evaluate EPIG-Seq's ability to extract biologically relevant patterns of gene expression, we downloaded count-level breast ductal carcinoma RNA-Seq data [17] produced on the Illumina GAII sequencer and processed as described in the Additional file 1. The RNA-Seq data was obtained from the specimens of patients with appropriate informed consent pre-existing with the TCGA repository. The breast tumor samples were classified by the mRNA subtypes [24-26]. We only used data from the following four subtypes: luminal A, luminal B, Her2enriched and basal-like. The latter subtype is often consider aggressive and to have a poorer prognosis. Patterns extracted with co-expressed genes exhibiting varied expression within the basal-like subtype apart from the other subtypes would be of interest for molecular profiling of the tumor. To generate 4 separate "sampled" data sets of reasonable size $(n=50)$, for each one, we randomly selected 10 lanes from each tumor subtype plus 10 lanes from normal breast tissue.

\section{Toxicogenomics RNA-Seq data}

The cascade of biochemical and molecular initiating events (MIEs; i.e., the biological targets of a chemical) following a toxicological exposure is referred to as the MOA. RNA-Seq data from the MAQC phase III SEQC crowd source toxicogenomics (TGxSEQC) effort [1] was acquired from the livers of male Sprague-Dawley rats exposed to chemicals that share a MOA and is available in the National Center for Biotechnology Information Sequence Read Archive (SRA) [27] under accession number SRP039021 and the Gene Expression Omnibus under accession number GSE55347. We used the training data set containing RNA-Seq data from 15 chemicals or vehicle and route matched controls. Animals were handled in accordance with United States Department of Agriculture and Code of Federal Regulations Animal Welfare Act (9 CFR Parts 1, 2, and 3). Sets of three chemicals share one of five MOAs. Three MOAs are associated with well-defined receptor-mediated processes-peroxisome proliferator-activated receptor alpha (PPARA), orphan nuclear hormone receptors (CAR/PXR) and aryl 
hydrocarbon receptor (AhR). The other two are nonreceptor-mediated-DNA damage (DNA_Damage) or cytotoxicity (Cytotoxic). Patterns extracted with genes exhibiting varied co-expression in one or more MOAs may elucidate MIEs shared between chemicals. Specific details of the study design and sample collection are available in the TGxSEQC publication. Further details of the alignment of the RNA-Seq reads and bioinformatics pipeline are available in the Additional file 1.

\section{Software usability}

Using EPIG-Seq to identify patterns of gene expression and to identify co-expressed gene is straight forward and simple using the graphical user interface (GUI). The data format for analysis requires a tab-delimited text file with the 1st row containing the labels of the groups $(n>3)$ that the samples belong to (one group must be of samples that are controls/baseline/background) and the 2nd row containing the total mapped reads for each sample. The latter is used for visualization of the results as log base 2 ratio reads per million (RPM) data. The $1^{\text {st }}$ column must contain unique gene IDs and the data in the remaining cells the read counts (as integers) per gene.

EPIG-Seq analysis proceeds in two steps (pattern identification and gene clustering), both of which have parameter setting for correlation of the genes and magnitude of differential gene expression change (in at least one group compared to the baseline/controls/background samples). Correlation is based on the $\mathrm{CY}_{\mathrm{s}}$ measure with a higher value denoting more correlation. Magnitude of differential change is according to the Z-statistics from the Wilcoxon rank sum non-parametric tests of each comparator group to the baseline/controls/background group. Thus, the magnitude of change resembles the deviation from a standard normal distribution. For instance, a Z-statistic $=2$ translates to an approximate probability of 0.05 that the gene expression is statistically different in the comparator group than the baseline/controls/background group. Since the $\mathrm{CY}_{\mathrm{s}}$ measure doesn't account for direct or anticorrelation, in EPIG-Seq the signs of the Z-statistics are used to constrain the directionality of the correlation.

Step 1 (pattern identification) has three additional user-defined parameters: 1) minimum number of gene profiles to make a pattern, 2) the correlation setting to weed out redundant patterns and 3) the number of central processing units (CPUs) to use for processing. Increasing the first two parameters will reduce the number of patterns extracted. Increasing the number of CPUs will substantially decrease the processing time as parallel computing is utilized. Lastly, step 1 has a gene dispersion threshold setting to discard gene profiles from pattern consideration if their dispersions are $<$ or $>5 \%$ in each tail of the distribution.
Step 2 (gene clustering) has an additional user-defined parameter for the number of bootstraps needed to compute the non-parametric $p$-value in determining significance of the patterns. Increasing the number of bootstraps will increase the reliability of the estimated $p$-value with a cost of a longer processing time. Finally, step 2 has a clustering iteration threshold equal to either a $<0.0001$ difference between two successive recursive clusterings of the genes to the patterns or the clustering recursion proceeded for 100 iterations.

The main steps in the EPIG-Seq analysis proceeds as follows:

- Compute Z-statistics for each gene profile

- Perform pairwise correlations between gene profiles

- Extract patterns based on Step 1 parameters

- Remove redundant patterns

- Use the gene profiles with the top PCS from each unique pattern as the seeds for the gene profile clustering

- Compute the $p$-value for each gene profile

- Terminate clustering of profiles to patterns

- Compute $p$-values for the patterns

- Present the results in output files, figures and a table of co-expressed genes

\section{Results}

\section{Development of EPIG-Seq}

We patterned the development of EPIG-Seq to resemble the steps and components that comprise EPIG [15] for analyzing gene expression patterns from microarray data. As shown in Table 1, EPIG-Seq uses a $C Y_{s}$ measure, the magnitude of a Wilcoxon rank sum statistic and variance to mean ratio (VMR) for RNA-Seq count data. These provide several advantages of EPIG-Seq on the analysis of RNA-Seq data. First, it supports cases where the read count is zero. Second, since correlation across samples is used, EPIG-Seq is not affected by differences in total read count per sample/lane of RNA-Seq. Third, it supports the discrete Poisson distribution typical of RNA-Seq count data and uses a quasi-Poisson model to account for dispersion in the data. Finally, when within group sample sizes are small, it uses the robust and nonparametric Hodges-Lehmann estimator as the location parameter for the magnitude of gene expression change.

Table 1 EPIG-Seq configuration

\begin{tabular}{lc}
\hline Data type & Count level \\
\hline Distribution assumption & Poisson \\
Correlation measurement & CYs \\
Spread of the data & Dispersion \\
Magnitude of change & Wilcoxon test Z-Statistic \\
Dynamic range & Variance-to-mean ratio (VMR) \\
\hline
\end{tabular}




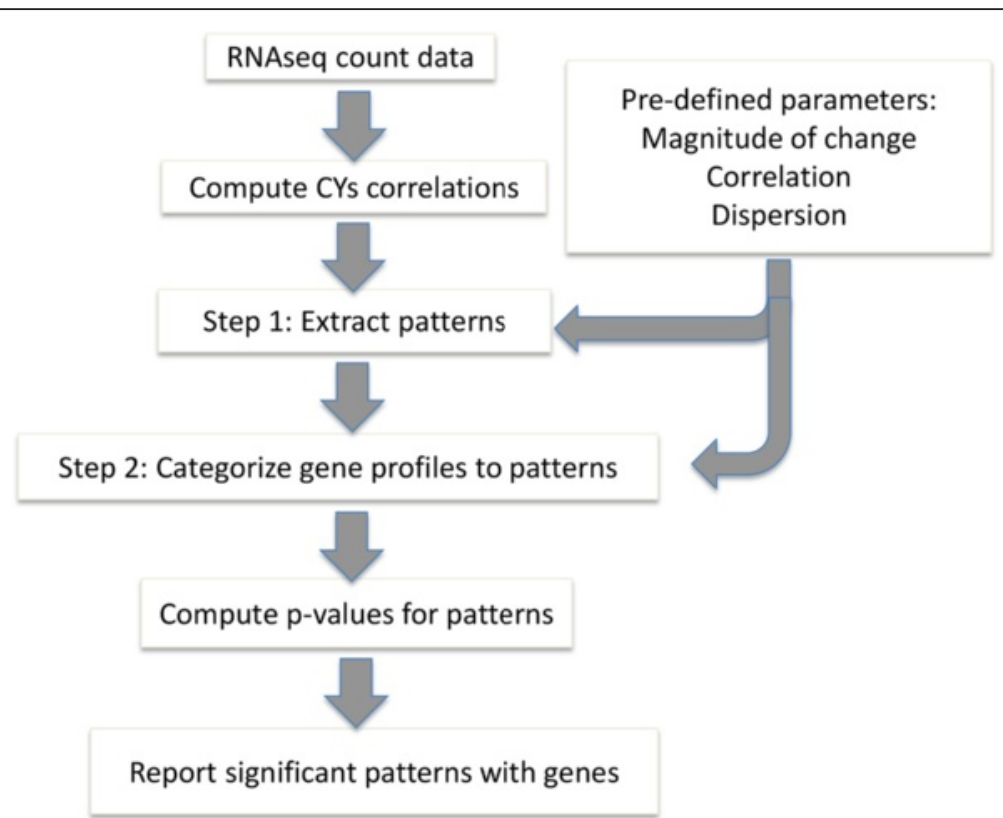

Fig. 1 EPIG-Seq workflow. The workflow depicts the main steps of EPIG-Seq. The parameters are used in steps 1 and 2 to extract the patterns and cluster the genes respectively. The output is the statistically significant patterns with co-expressed genes

As shown in Fig. 1 , the $1^{\text {st }}$ step in EPIG-Seq is to find all patterns in the data. Once the patterns are identified, in step 2, the gene expression profiles are clustered to the patterns to group co-expressed genes. Clustering is performed iteratively until the patterns with the coexpressed genes stabilize. The gene is assigned to the pattern to which it has the highest similarity to. Once all the genes are assigned, a representative gene for each of the patterns is determined by the PCS which is the highest median correlation to the other genes in the pattern.

Figure 2 shows the GUI for EPIG-Seq. Default values for the parameters are preassigned for pattern extraction

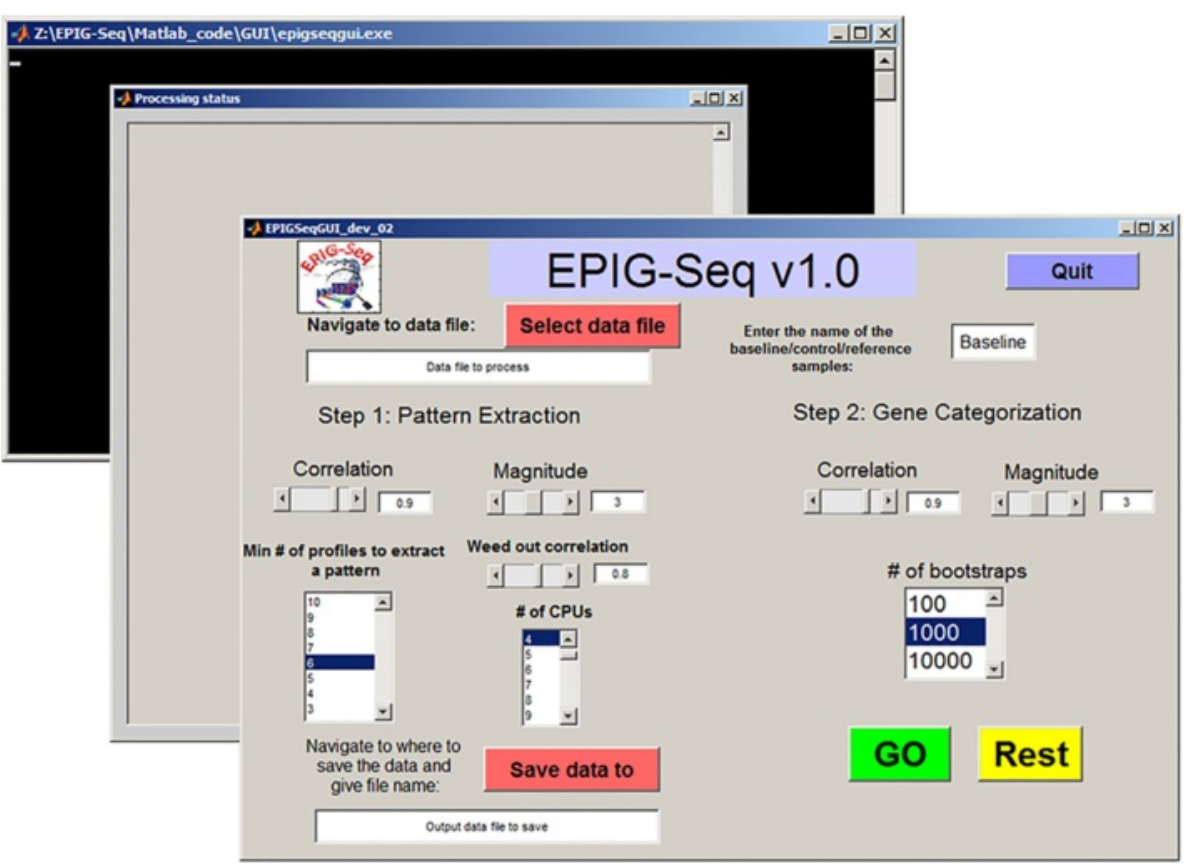

Fig. 2 EPIG-Seq GUI. The EPIG-Seq GUI contains a main panel which allows users to define parameters for steps 1 and 2 of the analysis process. A dialog box displays the processing status and a command window displays the dependent processes running in the background 


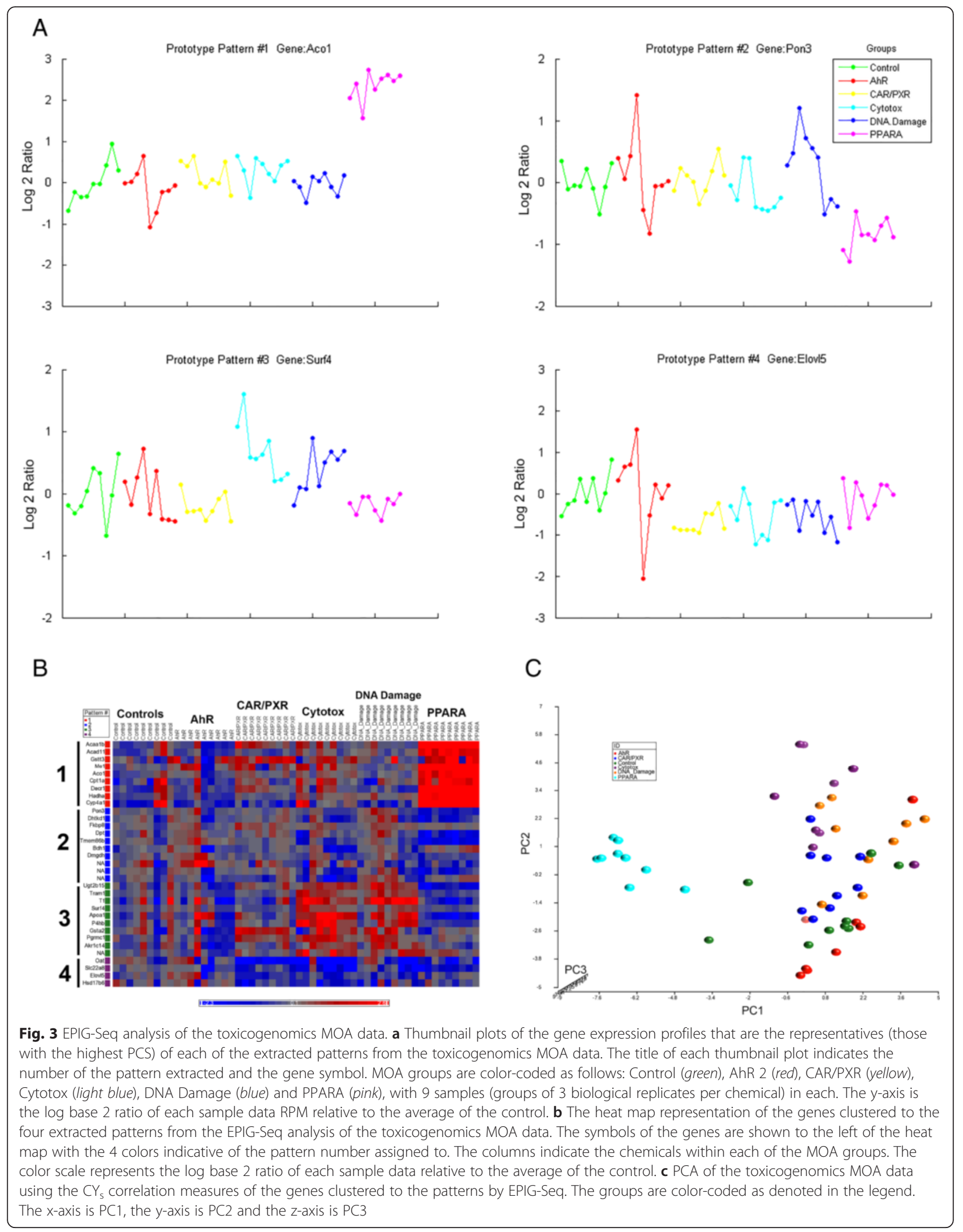


Table 2 Co-expressed genes from EPIG-Seq analysis of the MOA RNA-Seq data

\begin{tabular}{|c|c|c|c|}
\hline Pattern \# & Genebank Acc. \# & Symbol & Description \\
\hline 1 & NM_001040019 & Acaa1b & $\begin{array}{l}\text { Acetyl-Coenzyme A } \\
\text { acyltransferase 1B }\end{array}$ \\
\hline 1 & NM_001108181 & Acad11 & $\begin{array}{l}\text { Acyl-CoA dehydrogenase } \\
\text { family, member } 11\end{array}$ \\
\hline 1 & NM_001137643 & Gstt3 & $\begin{array}{l}\text { Glutathione S-transferase, } \\
\text { theta } 3\end{array}$ \\
\hline 1 & NM_012600 & Me1 & $\begin{array}{l}\text { Malic enzyme 1, NADP(+)- } \\
\text { dependent, cytosolic }\end{array}$ \\
\hline 1 & NM_017321 & Acol & Aconitase 1 , soluble \\
\hline 1 & NM_031559 & Cpt1a & $\begin{array}{l}\text { Carnitine palmitoyltransferase } \\
1 \mathrm{a} \text {, liver }\end{array}$ \\
\hline 1 & NM_057197 & Decr1 & $\begin{array}{l}\text { 2,4-dienoyl CoA reductase } 1 \text {, } \\
\text { mitochondrial }\end{array}$ \\
\hline 1 & NM_130826 & Hadha & $\begin{array}{l}\text { Hydroxyacyl-CoA } \\
\text { dehydrogenase/3-ketoacyl-CoA } \\
\text { thiolase/enoyl-CoA hydratase } \\
\text { (trifunctional protein), alpha } \\
\text { subunit }\end{array}$ \\
\hline 1 & NM_175837 & Cyp4a1 & $\begin{array}{l}\text { Cytochrome P450, family } 4 \text {, } \\
\text { subfamily a, polypeptide } 1\end{array}$ \\
\hline 2 & NM_001004086 & Pon3 & Paraoxonase 3 \\
\hline 2 & NM_001025720 & Dhtkd1 & $\begin{array}{l}\text { Dehydrogenase } \mathrm{E} 1 \text { and } \\
\text { transketolase domain } \\
\text { containing } 1\end{array}$ \\
\hline 2 & NM_001037180 & Fkbp8 & FK506 binding protein 8 \\
\hline 2 & NM_001105965 & Dpt & Dermatopontin \\
\hline 2 & NM_001109604 & Tmem86b & Transmembrane protein 86B \\
\hline 2 & NM_053995 & Bdh1 & $\begin{array}{l}\text { 3-hydroxybutyrate } \\
\text { dehydrogenase, type } 1\end{array}$ \\
\hline 2 & NM_139102 & Dmgdh & Dimethylglycine dehydrogenase \\
\hline 2 & XM_002728268 & NA & NA \\
\hline 2 & XM_002728512 & NA & NA \\
\hline 2 & XM_002728876 & NA & NA \\
\hline 3 & NM_001004271 & Ugt2b15 & $\begin{array}{l}\text { UDP glucuronosyltransferase } 2 \\
\text { family, polypeptide B15 }\end{array}$ \\
\hline 3 & NM_001007701 & Tram1 & $\begin{array}{l}\text { Translocation associated } \\
\text { membrane protein } 1\end{array}$ \\
\hline 3 & NM_001013110 & Tf & Transferrin \\
\hline 3 & NM_001033868 & Surf4 & Surfeit 4 \\
\hline 3 & NM_012738 & Apoa1 & Apolipoprotein A-I \\
\hline 3 & NM_012998 & P4hb & $\begin{array}{l}\text { Prolyl 4-hydroxylase, } \\
\text { beta polypeptide }\end{array}$ \\
\hline 3 & NM_017013 & Gsta2 & $\begin{array}{l}\text { Glutathione S-transferase } \\
\text { alpha } 2\end{array}$ \\
\hline 3 & NM_021766 & Pgrmc1 & $\begin{array}{l}\text { Progesterone receptor } \\
\text { membrane component } 1\end{array}$ \\
\hline 3 & NM_138547 & Akr1c14 & $\begin{array}{l}\text { Aldo-keto reductase family } 1 \text {, } \\
\text { member } \mathrm{C} 14\end{array}$ \\
\hline 3 & NM_175843 & NA & NA \\
\hline 4 & NM_022521 & Oat & Ornithine aminotransferase \\
\hline
\end{tabular}

Table 2 Co-expressed genes from EPIG-Seq analysis of the MOA RNA-Seq data (Continued)

\begin{tabular}{llll}
\hline 4 & NM_031332 & Slc22a8 & $\begin{array}{l}\text { Solute carrier family 22 } \\
\text { (organic anion transporter), } \\
\text { member } 8\end{array}$ \\
4 & NM_134382 & Elovl5 & ELOVL fatty acid elongase 5 \\
4 & NM_173305 & Hsd17b6 & $\begin{array}{l}\text { Hydroxysteroid (17-beta) } \\
\text { dehydrogenase 6 }\end{array}$ \\
\hline
\end{tabular}

(step 1) and gene clustering (step 2) but can be changed to suit the analysis stringency (see Additional file 2: Figure S1A and B for optimization of parameters for EPIG-Seq steps 1 and 2 respectively using simulated data). The five patterns extracted from the simulated data illustrate the utility of EPIG-Seq to extract only the real responsive patterns (not the noisy one), identify co-expressed genes and group the samples (Additional file 3: Figure S2A, B and C respectively). Increasing the correlation will result in fewer patterns extracted and fewer genes clustered. Increasing the magnitude will require larger fold change responses cross the treatment groups. Increasing the weed out correlation will result in fewer redundant patterns extracted. The status of the EPIG-Seq processing of the data is monitored through a dialog box.

\section{EPIG-Seq co-expression analysis}

To demonstrate the utility of EPIG-Seq, we analyzed real RNA-Seq data. EPIG-Seq analysis of the MAQC Toxicogenomics data set (samples of RNA from the livers of rats exposed to chemicals that share a common mode of action) extracted four patterns of coexpressed genes when using $C Y_{s}$ and $\mathrm{St}$ for steps 1 and 2 equal to 0.8 and 1 respectively and percentile $(\mathrm{PCT})=5 \%$ as parameter settings (Fig. 3a). The pattern representatives (genes used as seeds) are shown (Aco1, Pon3, Surf4 and Elovl5). The more impacting treatments in terms of gene regulation were seen from the exposure to PPARA chemicals (Fig. 3b). This biased coexpression of genes is expected as the chemicals with the PPARA MOA (Bezafibrate, Nafenopin and Pirinixic acid) were shown to have about $59 \%$ more differential expression $(\sim 6,500$ on average) than the chemicals in the other

Table $3 \mathrm{GO}$ biological processes of MOA clustered genes

\begin{tabular}{cclcc}
\hline Pattern \# & \# of Genes & Top GOBP & $p$-value & FDR \\
\hline 1 & 9 & $\begin{array}{l}\text { GO:0006631 - Fatty acid } \\
\text { metabolic process }\end{array}$ & $3.8 \mathrm{E}-06$ & $4.4 \mathrm{E}-03$ \\
2 & 10 & $\begin{array}{l}\text { GO:0055114 - Oxidation } \\
\text { reduction process }\end{array}$ & $2.3 \mathrm{E}-02$ & $2.1 \mathrm{E}+01$ \\
3 & 10 & $\begin{array}{l}\text { GO:0042592 - Homeostatic } \\
\text { process }\end{array}$ & $6.0 \mathrm{E}-02$ & $5.5 \mathrm{E}+01$ \\
& & & &
\end{tabular}

4

GOBP Gene Ontology biological processes filtered to remove very broad $\mathrm{GO}$ terms 
MOAs ( 4,100 on average) [1]. In other words, the PPARA MOA chemicals elicit a stronger transcriptional response than the other MOA chemicals. Also, despite the heterogeneity in the data and the three chemicals per MOA, EPIG-Seq was still able to extract discernable patterns. In pattern 1, the genes were upregulated by PPARA chemical treatments and unchanged by the other treatments. Pattern 2 depicts the converse. Pattern 3 shows a down-regulation by PPARA chemicals but a slight upregulation of genes by CAR/PXR, cytotoxic agents and DNA damage toxicants. Pattern 4 illustrates the downregulation of all the MOA chemicals except for AhR. There were 33 genes clustered in total (Table 2). Gene ontology biological processes enrichment of the genes reveals regulation of fatty acid metabolism, oxidation/reduction and homeostatic processes impacted by co-expressed genes in patterns 1, 2 and 3 respectively (Table 3 ). PCA of the 33 genes confirms that the treatments by the PPARA chemicals support the notion that the treated samples from the treatment of chemicals in this MOA are very different from the others (Fig. 3c).

As another example of EPIG-Seq's utility, we analyzed TCGA breast cancer RNA-seq data derived from 10 randomly selected lanes from each "subtype" to construct balanced data sets of the four breast cancer subtypes plus normal breast tissue as a control. Using $C Y_{s}$ and St for steps 1 and 2 equal to 0.8 and 2 respectively and PCT $=5 \%$ as parameter settings, EPIG-seq extracted four or six patterns from either of the TCGA sampled data with between 192 and 344 genes in total per sampled set (Table 4 and Additional file 4: Tables S1-S4). The general silhouette of the genes reveals that the patterns are relatively cohesive and separated well. Since the data sets were randomly selected from the pool, there is stochastic variation in the data that introduces variability in the results. From the clustering comparison (Table 5), good adjusted mutual information (AMI) agreement was observed, although comparisons between data set 1 and data sets 3 and 4 yielded low scores of 0.524 and 0.452 respectively. This points to the possibility that sampled data set 1 is somewhat of an outlier.

\section{Discussion}

RNA-Seq has its advantages over microarray gene expression analysis. Tools for analysis of RNA-Seq data

Table 4 EPIG-Seq clustering cohesiveness of patterns extracted from the TCGA breast cancer sampled data

\begin{tabular}{lcccc}
\hline Sample \# & GS & MS & \# of Patterns & \# of Genes \\
\hline 1 & 0.31 & 0.54 & 6 & 192 \\
2 & 0.37 & 0.51 & 4 & 169 \\
3 & 0.21 & 0.52 & 6 & 344 \\
4 & 0.41 & 0.59 & 4 & 197 \\
\hline
\end{tabular}

GS general silhouette, MS Maximal silhouette
Table 5 Agreement of clusters extracted from the TCGA breast cancer sampled data

\begin{tabular}{lc}
\hline Samples compared & Agreement \\
\hline 1 vs 2 & 0.770 \\
1 vs 3 & 0.524 \\
1 vs 4 & 0.452 \\
2 vs 3 & 0.691 \\
2 vs 4 & 0.751 \\
3 vs 4 & 0.500 \\
\hline
\end{tabular}

All comparisons based on AMl except for those with sample 2 where concordance was used

primarily test pair-wise comparisons or are analysis of variance (ANOVA)-like but they compute on the count data. We developed a version of our EPIG tool for microarray gene expression to support RNA-Seq count data (EPIG-Seq). An advantage of EPIG-Seq is that gene expression profiles from the RNA-Seq data are analyzed across a set of treatment conditions or series of perturbations. In addition, EPIG-Seq supports data with inflated zeros and that is overdispersed. Using countbased correlation to measure similarity between gene expression profiles, quasi-Poisson modelling to estimate dispersion in the data and a location parameter to indicate the strength of differential expression, EPIG-Seq clustered genes to the statistically significant patterns that they correlate with across conditions. Other tools for analysis of RNA-Seq count data are not directly comparable to EPIG-Seq since they don't correlate gene expression across the treatment $[4,11,12,28]$.

Analysis of EPIG-Seq on real data yielded biologically meaningful results about the co-regulation of genes. For example, analysis of the MAQC Toxicogenomics data set

Table 6 Pathway enrichment of breast cancer co-expressed genes

\begin{tabular}{|c|c|c|c|c|}
\hline Pattern \# & $\begin{array}{l}\text { \# of } \\
\text { Genes }\end{array}$ & $\begin{array}{l}\text { Enriched Pathways (example } \\
\text { of co-expressed genes) }\end{array}$ & $p$-value & FDR \\
\hline 1 & 45 & $\begin{array}{l}\text { GO:0006260 - DNA replication } \\
\text { (PCNA, TOP2A, S100A14) }\end{array}$ & $1.10 \mathrm{E}-05$ & $1.70 \mathrm{E}-02$ \\
\hline 2 & 182 & $\begin{array}{l}\text { GO:0006886 - Intracellular protein } \\
\text { transport (ERBB3, PTMS, SLC25A5, } \\
\text { SLC9A3R1, SOX4, STAT1) }\end{array}$ & 1.1. E-06 & $1.8 \mathrm{E}-03$ \\
\hline 3 & 9 & $\begin{array}{l}\text { BST2, C17orf37, CEACAM6, IFI27, } \\
\text { IFI6, MX1, OAS3, RAB31, TPX2 }\end{array}$ & - & - \\
\hline 4 & 40 & $\begin{array}{l}\text { KEGG:03320 - Peroxisome proliferator- } \\
\text { activated receptor signaling pathway } \\
\text { (TRIM29, PDK4, FOSB, CD36) }\end{array}$ & $1.0 \mathrm{E}-04$ & $9.1 \mathrm{E}-02$ \\
\hline 5 & 41 & $\begin{array}{l}\text { GO:0010033 - Response to organic } \\
\text { substance (ANXA1, CD34, EGR1, FOS, } \\
\text { TGFBR2) }\end{array}$ & 1.3E-04 & $2.0 \mathrm{E}-01$ \\
\hline 6 & 27 & $\begin{array}{l}\text { GO:0006414 - Translation elongation } \\
\text { (CD59, ITGB1, ribosomal protein genes) }\end{array}$ & 2.80E-05 & $3.90 \mathrm{E}-02$ \\
\hline
\end{tabular}


with RNA samples of livers from rats exposed to chemicals that share a MOA, EPIG-Seq identified genes in patterns that are key toxicological processes in metabolism and oxidation/reduction (Table 3). For instance, in pattern 1 where the genes are up-regulated by the PPARA MOA chemicals and essentially unchanged in the other treatments (Fig. 3a and b), Cytochrome P450, Family 4,
Subfamily A, Polypeptide 1 (Cyp4a1 -PPARA inducible) is one of the hallmark PPARA responsive genes coexpressed [29]. In the rat liver, Cyp4a1 is induced by binding of peroxisome proliferator ligands to the PPARA receptor [30]. Furthermore, motif analysis [31] of the -1000 to +1000 DNA sequence region of the nine genes in pattern 4 uncovered an enriched

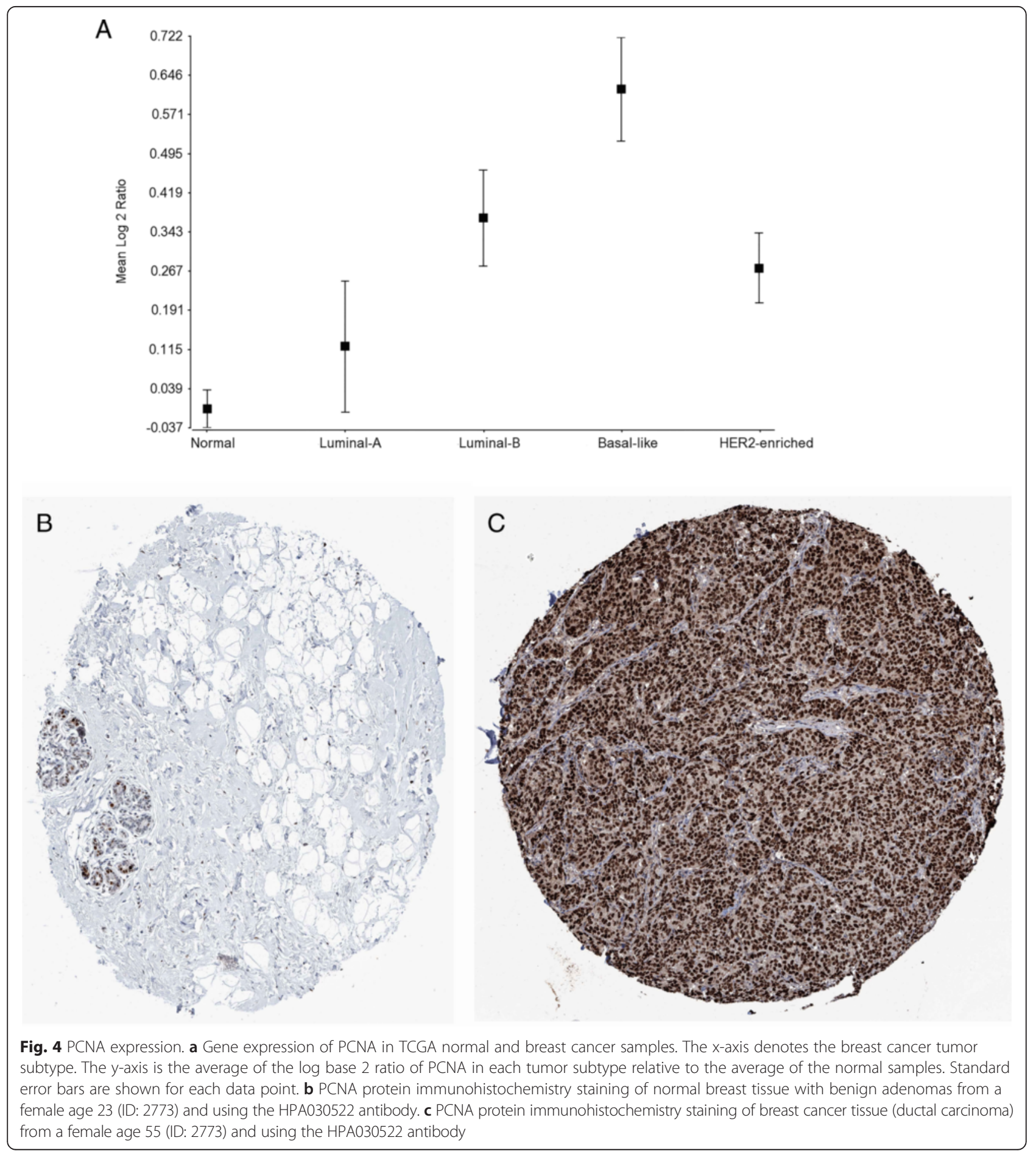


transcription factor binding site (TATAACA) as overrepresented with a $p$-value $=4.17 \times 10^{-4}$.

Analysis of the TCGA breast cancer data sample \#3 produced DNA replication, intracellular protein transport, PPAR signaling, response to organic substances and translation elongation biological pathways as overrepresented categories (Table 6) commonly associated with breast cancer progression and metastasis [32, 33]. In particular, pattern \#1 contains the up-regulation of proliferating cell nuclear antigen (PCNA) (Fig. 4a), topoisomerase (DNA) II (TOP2A) and S100 calcium binding protein A14 (S100A14) genes. In fact, PCNA, TOP2A and other genes in the patterns have been targets for breast cancer therapy [34,35]. Interestingly, the Human Protein Atlas [36] contains immunohistochemistry staining of the PCNA protein (antibody HPA030522) in normal breast tissue (Fig. 4b) versus the overexpression in breast cancer ductal carcinoma (Fig. 4c) implicating the abundance of the protein in breast cancer tissue as a potential biomarker [37].

\section{Conclusions}

EPIG-Seq is unlike any other software currently available for pattern analysis of RNA-Seq count level data across experimental groups. EPIG-Seq analysis of RNA-Seq count data across biological conditions permits the ability to extract biologically meaningful co-expressed genes associated with coordinated regulation. The approach leverages a count based correlation to identify patterns of expression across samples, accounts for the dispersion in the data and uses a location parameter to indicate magnitude of differential expression whether the sample size is large or small. EPIG-Seq analysis of TCGA human breast cancer RNA-Seq data extracts genes regulated across the various subtypes including PCNA, one of the key marker genes. EPIG-Seq analysis of a rat liver toxicogenomics RNA-Seq data set reveals genes that coexpressed across MOAs containing chemicals with similar MIEs such as PPAR antagonists and the Cyp4a1 PPAR- $\alpha$ inducible gene. Thus, using the EPIG-Seq software to analyze RNA-Seq count data across biological conditions permits the ability to extract biologically meaningful co-expressed genes associated with coordinated regulation.

\section{Availability and requirements}

Project name: EPIG-Seq

Project home page: e.g. http://sourceforge.net/projects/ epig-seq

Operating system(s): Windows and Linux

CPU architecture: Multicores recommended

Programming language: e.g. $\mathrm{C}$ and Java currently, $\mathrm{R}$ in future implementations
Other requirements: $R$ version $\geq 3.1 .2$ and CRAN $R$ package stats version 3.1.2 to fit a generalized linear model (glm)

License: GNU GPL-2 | GPL-3

Any restrictions to use by non-academics: License needed to distribute the programs containing code from $\mathrm{R}$ and for the Matlab MCRInstaller from MathWorks.

\section{Additional files}

Additional file 1: Supplemental methods are more detailed descriptions of some of the computational methods used in EPIG-Seq. (DOCX 97 kb)

Additional file 2: Figure S1. $A$ and $B$ are tiff image files of the plots illustrating the optimization of parameters for EPIG-Seq steps 1 and 2 respectively using simulated data. (TIF 19517 kb)

Additional file 3: Figure S2. EPIG-Seq analysis of the simulated data. A) Thumbnail plots of the simulated gene profiles that are the representatives (those with the highest pattern correlation score) of each of the extracted patterns. B) Heat map representation of the patterns extracted and the simulated gene profiles clustered to each. C) PCA of the simulated gene profiles clustered to the patterns by EPIG-Seq using the CYs correlation measures. (JPG $3477 \mathrm{~kb}$ )

Additional file 4: Tables S1-S4. Are Excel spreadsheets containing the EPIG-Seq clustered genes from the TCGA data samples 1 - 4 respectively. (XLSX $665 \mathrm{~kb})$

\section{Abbreviations}

AhR: aryl hydrocarbon receptor; AMl: adjusted mutual information; ANOVA: analysis of variance; ARI: adjusted Rand Index; CAR/PXR: Constitutive Androstane Receptor/Pregnane X Receptor; CPUs: central processing units; Cyp4a1: Cytochrome P450, Family 4, Subfamily A, Polypeptide 1;

$\mathrm{CY}_{\text {s: }}$ correlation for count data; Cytotoxic: cytotoxicity; DNA_Damage: DNA damage; EPIG: extracting patterns and identifying co-expressed genes; EPIG-Seq: extracting patterns and identifying co-expressed genes from RNA-Seq data; GUI: graphical user interface; MAQC: MicroArray Quality Control; MI: mutual information; MIE: molecular initiating event; MOA: mode of action; Mt: number of profiles to form candidate patterns in EPIG-Seq step 1; NB: negative binomial; NCl: National Cancer Institute; PCA: principal component analysis; PCNA: proliferating cell nuclear antigen; PCS: pattern correlation score; PCT: percentile; PPARA: peroxisome proliferator-activated receptor alpha; RNA-Seq: RNA sequencing; RPM: reads per million; Rt1: $C Y_{s}$ correlation for EPIG-Seq step 1; Rt2: $\mathrm{CY}_{\mathrm{s}}$ correlation for EPIG-Seq step 2; SEQC: SEquence Quality Control; SNR: signal to noise ratio; SRA: sequence read archive; St1: magnitude of fold change for EPIG-Seq step 1;

St2: magnitude of fold change for EPIG-Seq step 2; TCGA: The Cancer Genome Atlas; TGXSEQC: Toxicogenomics SEQC; TOP2A: Topoisomerase (DNA) II; VMR: variance to mean ratio.

\section{Competing interests}

The authors declare that they have no competing interests.

\section{Authors' contributions}

PRB conceived of the EPIG-Seq methodology. JL and PRB developed the EPIG-Seq methodology, obtained the data, performed the analyses and drafted the manuscript. PRB implemented the EPIG-Seq software. All authors read and approved the final manuscript.

\section{Acknowledgements}

We thank the MicroArray Quality Control phase III (MAQC-III) Sequence Quality Control (SEQC) toxicogenomics working group members (TGXSEQC) Weida Tong, Charles Wang, Joshua Xu, Binsheng Gong and Zhenqiang Su for contributing the RNA-Seq mapped reads from the raw Toxicogenomics MOA data. We thank Raja Jothi and Oswaldo A. Lozoya for their critical review of the paper. The results published here are in whole or part based upon data generated by TCGA pilot project established by the $\mathrm{NCl}$ and NHGRI. Information about TCGA and the investigators and institutions who 
constitute the TCGA research network can be found at http://cancergenome. nih.gov. We acknowledge Jeff Chou for his insight to the details of EPIG and Joel Parker for help with obtaining the TCGA breast cancer subtype information. This research was supported, in part, by the Intramural Research Program of the National Institutes of Health $(\mathrm{NIH})$, National Institute of Environmental Health Sciences (NIEHS)

\section{Author details}

${ }^{1}$ Integrative Bioinformatics Group, National Institute of Environmental Health Sciences, Research Triangle Park, NC 27709, USA. ${ }^{2}$ Microarray and Genome Informatics Group, National Institute of Environmental Health Sciences, Research Triangle Park, NC 27709, USA. ${ }^{3}$ Biostatistics and Computational Biology Branch, National Institute of Environmental Health Sciences, 111 T.W. Alexander Drive, P.O. Box 12233, Research Triangle Park, NC 27709, USA. ${ }^{4}$ Kelly Government Solutions, Research Triangle Park, NC 27709, USA.

\section{Received: 30 December 2015 Accepted: 11 March 2016}

\section{Published online: 22 March 2016}

\section{References}

1. Wang C, Gong B, Bushel PR, Thierry-Mieg J, Thierry-Mieg D, Xu J, Fang H, Hong $\mathrm{H}$, Shen J, Su Z, et al. The concordance between RNA-seq and microarray data depends on chemical treatment and transcript abundance. Nat Biotechnol. 2014;32(9):926-32

2. Merrick BA, Phadke DP, Auerbach SS, Mav D, Stiegelmeyer SM, Shah RR, Tice RR. RNA-Seq profiling reveals novel hepatic gene expression pattern in aflatoxin B1 treated rats. PLOS ONE. 2013;8(4):e61768.

3. Raghavachari N, Barb J, Yang Y, Liu P, Woodhouse K, Levy D, O'Donnell CJ, Munson PJ, Kato GJ. A systematic comparison and evaluation of high density exon arrays and RNA-seq technology used to unravel the peripheral blood transcriptome of sickle cell disease. BMC Med Genet. 2012;5:28.

4. Anders S, Huber W. Differential expression analysis for sequence count data. Genome Biol. 2010;11(10):R106.

5. Robinson MD, McCarthy DJ, Smyth GK. edgeR: a Bioconductor package for differential expression analysis of digital gene expression data. Bioinformatics. 2010;26(1):139-40.

6. Zhou YH, Xia K, Wright FA. A powerful and flexible approach to the analysis of RNA sequence count data. Bioinformatics. 2011;27(19):2672-8.

7. Zyprych-Walczak J, Szabelska A, Handschuh L, Gorczak K, Klamecka K, Figlerowicz M, Siatkowski I. The impact of normalization methods on RNA-Seq data analysis. BioMed Res Int. 2015;2015:621690.

8. Vijay N, Poelstra JW, Kunstner A, Wolf JB. Challenges and strategies in transcriptome assembly and differential gene expression quantification. A comprehensive in silico assessment of RNA-seq experiments. Mol Ecol. 2013;22(3):620-34

9. Dillies MA, Rau A, Aubert J, Hennequet-Antier C, Jeanmougin M, Servant N, Keime C, Marot G, Castel D, Estelle J, et al. A comprehensive evaluation of normalization methods for Illumina high-throughput RNA sequencing data analysis. Brief Bioinform. 2013;14(6):671-83.

10. O'Hara RB, Kotze DJ. Do not log-transform count data. Methods Ecol Evol. 2010;1(2):118-22.

11. Li J, Tibshirani R. Finding consistent patterns: a nonparametric approach for identifying differential expression in RNA-Seq data. Stat Methods Med Res. 2013;22(5):519-36.

12. Li J, Witten DM, Johnstone IM, Tibshirani R. Normalization, testing, and false discovery rate estimation for RNA-sequencing data. Biostatistics. 2012;13(3):523-38.

13. Lund SP, Nettleton D, McCarthy DJ, Smyth GK. Detecting differential expression in RNA-sequence data using quasi-likelihood with shrunken dispersion estimates. Stat Appl Genet Mol Biol. 2012;11(5). doi:10.1515/1544-6115.1826.

14. Spies D, Ciaudo C. Dynamics in transcriptomics: advancements in RNA-seq time course and downstream analysis. Comput Struct Biotechnol J. 2015;13:469-77.

15. Chou JW, Zhou T, Kaufmann WK, Paules RS, Bushel PR. Extracting gene expression patterns and identifying co-expressed genes from microarray data reveals biologically responsive processes. BMC Bioinformatics. 2007:8:427.

16. Cao Y, Williams WP, Bark AW. Similarity measure bias in River Benthic Aufwuchs Community analysis. Water Environ Res. 1997;69(1):95-106.

17. Cancer Genome Atlas Network. Comprehensive molecular portraits of human breast tumours. Nature. 2012;490(7418):61-70.
18. Horn HS. Measurement of overlap in comparative ecological studies. Am Nat. 1966:100(914):419-24.

19. Anderson MJ, Thompson AA. Multivariate control charts for ecological and environmental monitoring. Ecological Applications. 2004;14(6):1921-35.

20. Hollander MW, Douglas A, Chicken E. Nonparametric Statistical Methods. Hoboken: John Wiley \& Sons; 2013.

21. McCullagh P, Nelder JA. Generalized linear models. 2nd ed. Boca Raton: Chapman \& Hall/CRC; 1998.

22. Romesburg HC. Cluster analysis for researchers. Belmont: Lifetime Learning Publications; 1984

23. Kaufman L, Rousseeuw PJ. Finding groups in data : an introduction to cluster analysis. New York: Wiley; 1990.

24. Perou CM, Sorlie T, Eisen MB, van de Rijn M, Jeffrey SS, Rees CA, Pollack JR, Ross DT, Johnsen $H$, Akslen LA, et al. Molecular portraits of human breast tumours. Nature. 2000:406(6797):747-52.

25. Sorlie T, Perou CM, Tibshirani R, Aas T, Geisler S, Johnsen H, Hastie T, Eisen MB, van de Rijn M, Jeffrey SS, et al. Gene expression patterns of breast carcinomas distinguish tumor subclasses with clinical implications. Proc Natl Acad Sci U S A. 2001;98(19):10869-74.

26. Parker JS, Mullins M, Cheang MC, Leung S, Voduc D, Vickery T, Davies S, Fauron $C_{1}, \mathrm{He} X_{1} \mathrm{Hu}$ Z, et al. Supervised risk predictor of breast cancer based on intrinsic subtypes. J Clin Oncol. 2009:27(8):1160-7.

27. Wheeler DL, Barrett T, Benson DA, Bryant SH, Canese K, Chetvernin V, Church DM, Dicuccio M, Edgar R, Federhen S, et al. Database resources of the National Center for Biotechnology Information. Nucleic Acids Res. 2008:36(Database issue):D13-21.

28. Peddada S, Harris S, Zajd J, Harvey E. ORIOGEN: order restricted inference for ordered gene expression data. Bioinformatics. 2005;21(20):3933-4.

29. Alvergnas M, Richert L, Blanchard N, Abadie C, Heyd B, Mantion G, Gallemann D, Martin H. Regulation of CYP4A expression by bezafibrate in primary culture of rat and human hepatocytes: interspecies difference and influence of $\mathrm{N}$-acetylcysteine. Toxicol in Vitro. 2009;23(7):1259-67.

30. Casarett $\sqcup$, Doull J, Klaassen CD. Casarett and Doull's toxicology : the basic science of poisons. 6th ed. New York: McGraw-Hill Medical Pub. Division; 2001

31. Elemento O, Slonim N, Tavazoie S. A universal framework for regulatory element discovery across all genomes and data types. Mol Cell. 2007:28(2):337-50

32. Oskarsson T. Extracellular matrix components in breast cancer progression and metastasis. Breast. 2013;22 Suppl 2:S66-72.

33. Suchanek KM, May FJ, Robinson JA, Lee WJ, Holman NA, Monteith GR, Roberts-Thomson SJ. Peroxisome proliferator-activated receptor alpha in the human breast cancer cell lines MCF-7 and MDA-MB-231. Mol Carcinog. 2002;34(4):165-71.

34. Yu YL, Chou RH, Liang JH, Chang WJ, Su KJ, Tseng YJ, Huang WC, Wang SC, Hung MC. Targeting the EGFR/PCNA signaling suppresses tumor growth of triple-negative breast cancer cells with cell-penetrating PCNA peptides. PLOS ONE. 2013;8(4):e61362.

35. Romero A, Martin M, Cheang MC, Lopez Garcia-Asenjo JA, Oliva B, He X de la Hoya M, Garcia Saenz JA, Arroyo Fernandez M, Diaz Rubio E, et al. Assessment of Topoisomerase II alpha status in breast cancer by quantitative PCR, gene expression microarrays, immunohistochemistry, and fluorescence in situ hybridization. Am J Pathol. 2011;178(4):1453-60.

36. Ponten F, Jirstrom K, Uhlen M. The Human Protein Atlas-a tool for pathology. J Pathol. 2008;216(4):387-93

37. Malkas LH, Herbert BS, Abdel-Aziz W, Dobrolecki LE, Liu Y, Agarwal B, Hoelz D, Badve S, Schnaper L, Arnold RJ, et al. A cancer-associated PCNA expressed in breast cancer has implications as a potential biomarker. Proc Natl Acad Sci U S A. 2006;103(51):19472-7. 